\title{
AN OBSTRUCTION FOR THE MEAN CURVATURE OF A CONFORMAL IMMERSION $S^{n} \rightarrow \mathbb{R}^{n+1}$
}

\author{
BERND AMMANN, EMMANUEL HUMBERT, AND MOHAMEDEN OULD AHMEDOU
}

(Communicated by Mikhail Shubin)

\begin{abstract}
We prove a Pohozaev type identity for non-linear eigenvalue equations of the Dirac operator on Riemannian spin manifolds with boundary. As an application, we obtain that the mean curvature $H$ of a conformal immersion $S^{n} \rightarrow \mathbb{R}^{n+1}$ satisfies $\int \partial_{X} H=0$ where $X$ is a conformal vector field on $S^{n}$ and where the integration is carried out with respect to the Euclidean volume measure of the image. This identity is analogous to the Kazdan-Warner obstruction that appears in the problem of prescribing the scalar curvature on $S^{n}$ inside the standard conformal class.
\end{abstract}

Let $(M, g)$ be a compact Riemannian manifold with a conformal vector field $X$. Given a function $s$ on $M$, then it is a classical question to ask whether $s$ is the scalar curvature of a metric $\tilde{g}$ conformal to $g$. The determination of the set of all such functions $s$ is still open, although several partial results are known; in particular there are necessary conditions that $s$ has to satisfy in order to be a scalar curvature.

On the one hand there are topological obstructions. If for example $M$ is spin and has non-vanishing $\hat{A}$ genus, then the scalar curvature of any metric on $M$ has either to be negative somewhere or the Ricci curvature vanishes everywhere on $M$.

However, if one fixes the conformal class $[g]$ as described above, there are further obstructions that arise from conformal vector fields. For example if $M$ is $S^{n}$ with the standard conformal structure, Kazdan and Warner [KW75] derived a famous obstruction. A slightly stronger version of this obstruction due to Bourguignon and Ezin [BE87] is described in the following theorem.

Theorem 1. Let $X$ be a conformal vector field on the compact manifold $(M, g)$. If $s$ is the scalar curvature of a metric $\tilde{g}=u^{4 /(n-2)} g$, then

$$
\int_{M} \partial_{X} s d v_{\tilde{g}}=0
$$

where $d v_{\tilde{g}}=u^{\frac{2 n}{n-2}} d v_{g}$ is the volume measure associated to $\tilde{g}$.

Tightly related to the Kazdan-Warner obstruction is the Pohozaev identity. Let $\Omega$ be a star-shaped open set of $\mathbb{R}^{n}(n \in \mathbb{N})$ with smooth boundary. We denote by $\Delta=-\sum_{i=1}^{n} \partial_{i i}$ the Laplacian on $\mathbb{R}^{n}$. Let $u \in C^{2}(\bar{\Omega})$ be a positive solution of $\Delta u=u^{p-1}$ on $\Omega$ with $u_{\mid \partial \Omega} \equiv 0$. The vector field $X=\sum_{i=1}^{n} x^{i} \partial_{i}$ is conformal. If one uses similar methods as in the proof of the Kazdan-Warner obstruction, then

Received by the editors June 28, 2005 and, in revised form, September 6, 2005.

2000 Mathematics Subject Classification. Primary 53A27, 53A30, 35J60.

(C)2006 American Mathematical Society Reverts to public domain 28 years from publication 
one obtains the Pohozaev identity ([Po65] $)$ which asserts that

$$
\left(1-\frac{n}{2}+\frac{n}{p}\right) \int_{\Omega} u^{p}=\frac{1}{2} \int_{\partial \Omega}\langle\nu, X\rangle\left(\partial_{\nu} u\right)^{2},
$$

where $\nu$, resp. $\partial_{\nu}$, is the outer normal vector, resp. the outer normal derivative, on $\partial \Omega$. One among many important consequences of this inequality is that no non-trivial solutions exist if $p \geq \frac{2 n}{n-2}$. Another application is an alternative proof of the Kazdan-Warner obstructions in the case that $(M, g)$ is the sphere with the standard conformal structure [DR99.

In the present short article, we establish a similar identity for the classical Dirac operator $D$. We derive this identity on manifolds with boundary in order to admit future Pohozaev type applications. Then, we will specialize to compact manifolds without boundary, where we will derive a Kazdan-Warner type obstruction for the mean curvature of a conformal immersion $S^{2} \rightarrow \mathbb{R}^{3}$.

Our main theorem is:

Theorem 2. Let $(M, g, \chi)$ be a compact Riemannian spin manifold of dimension $n$ with boundary $\partial M$ (possibly equal to $\emptyset$ ) and with Dirac operator D. We assume that there exists a smooth spinor field $\psi$ which satisfies for some $p>1$,

$$
D \psi=H|\psi|^{p-2} \psi, \quad H \in C^{\infty}(M) .
$$

Furthermore, we assume that $X$ is a conformal vector field on $M$. Then, we have the following Pohozaev type identity:

$$
\begin{aligned}
\int_{\partial M}\left\langle\nu \cdot \mathcal{L}_{X} \psi, \psi\right\rangle= & \frac{p-2}{p} \int_{\partial M} H|\psi|^{p} g(X, \nu) \\
& +\left(\frac{1}{n}-\frac{p-2}{p}\right) \int_{M} H \operatorname{div} X|\psi|^{p}+\frac{2}{p} \int_{M}\left(\partial_{X} H\right)|\psi|^{p},
\end{aligned}
$$

where $\nu$ denotes the outward pointing normal vector along $\partial M$, and where $\langle$, denotes the real scalar product on spinors.

Proof. The flow associated to the conformal vector field $X$ will be denoted as $\alpha^{t}$. If $p$ is in the interior of $M$, then $\alpha^{t}(p)$ exists for times $t$ close to 0 . For any $t \in \mathbb{R}$ let $f^{t}$ be the conformal scaling function of $\alpha^{t}$, i.e. $\left(d \alpha^{t}\right)_{p}$ is $f^{t}(p)$ times an isometry from $T_{p} M$ to $T_{\alpha^{t}(p)} M$. Let $\alpha_{*}^{t}: \Sigma_{p} M \rightarrow \Sigma_{\alpha^{t}(p)} M$ be the spinor identification map as constructed in [Ht74, Hi86, BG92]. In particular, this map has the pointwise properties that

$$
\left|\alpha_{*}^{t}(\psi)\right|=|\psi|
$$

and the following transformation formula for conformal changes of the metric. Let $\varphi \in \Gamma(\Sigma M)$ be a spinor field. For $t$ close to 0 , we then define the map $\alpha_{\#}^{t}: \Gamma(\Sigma M) \rightarrow \Gamma(\Sigma \tilde{M}), \alpha_{\#}^{t}(\varphi):=\alpha_{*}^{t} \circ \varphi \circ \alpha^{-t}$, where $\tilde{M}$ is $M$ without an open neighborhood of the boundary.

Then

$$
D \alpha_{\#}^{t}\left(\left(f^{t}\right)^{-\frac{n-1}{2}} \psi\right)=\alpha_{\#}^{t}\left(\left(f^{t}\right)^{-\frac{n+1}{2}} D \psi\right) .
$$

Now we assume that $\psi$ satisfies (2), and we obtain

$$
D \alpha_{\#}^{t}\left(\left(f^{t}\right)^{-\frac{n-1}{2}} \psi\right)=\alpha_{\#}^{t}\left(\left(f^{t}\right)^{-\frac{n+1}{2}} H|\psi|^{p-2} \psi\right) .
$$


Differentiating with respect to $t$ at $t=0$ yields

$$
\begin{array}{r}
-\frac{n-1}{2} D \beta \psi+\left.D \frac{d}{d t}\right|_{t=0} \alpha_{\#}^{t} \psi=-\frac{n+1}{2} H \beta|\psi|^{p-2} \psi+\left.H|\psi|^{p-2} \frac{d}{d t}\right|_{t=0} \alpha_{\#}^{t} \psi \\
+(p-2) H\left\langle\left.\frac{d}{d t}\right|_{t=0} \alpha_{\#}^{t} \psi, \psi\right\rangle|\psi|^{p-4} \psi-\left(\partial_{X} H\right)|\psi|^{p-2} \psi
\end{array}
$$

where $\beta:=\left.\frac{d}{d t}\right|_{t=0} f^{t}$. We reformulate using the definition of the Lie derivative of spinor fields in the direction $X$ [BG92], i.e.

$$
\mathcal{L}_{X}(\psi)=-\left.\frac{d}{d t}\right|_{t=0} \alpha_{\#}^{t}(\psi)
$$

Together with $D \beta \psi=\beta D \psi+\nabla \beta \cdot \psi$ and (2) one then concludes that

$$
\begin{aligned}
& \frac{n-1}{2} \nabla \beta \cdot \psi+D \mathcal{L}_{X} H \beta|\psi|^{p-2} \psi+H|\psi|^{p-2} \mathcal{L}_{X} \psi \\
& \quad+(p-2) H\left\langle\mathcal{L}_{X} \psi, \psi\right\rangle|\psi|^{p-4} \psi+\left(\partial_{X} H\right)|\psi|^{p-2} \psi .
\end{aligned}
$$

After multiplication with $\psi$, the $\nabla \beta \cdot \psi$-term vanishes, and we obtain

$$
\left\langle D \mathcal{L}_{X} \psi, \psi\right\rangle=(p-1) H|\psi|^{p-2}\left\langle\mathcal{L}_{X} \psi, \psi\right\rangle+H \beta|\psi|^{p}+\left(\partial_{X} H\right)|\psi|^{p} .
$$

The product rule for the Lie derivative tells us that

$$
|\psi|^{p-2}\left\langle\mathcal{L}_{X} \psi, \psi\right\rangle=\frac{1}{2}|\psi|^{p-2} \partial_{X}|\psi|^{2}=|\psi|^{p-1} \partial_{X}|\psi|=\frac{1}{p} \partial_{X}|\psi|^{p}
$$

Hence, we obtain

$$
\left\langle D \mathcal{L}_{X} \psi, \psi\right\rangle=\frac{p-1}{p} H \partial_{X}|\psi|^{p}+H \beta|\psi|^{p}+\left(\partial_{X} H\right)|\psi|^{p} .
$$

Strictly speaking, this equation is valid in the interior, but it extends to the boundary by continuity. Now, we integrate over $M$. With partial integration for the Dirac operator one obtains

$$
\begin{aligned}
\int_{M}\left\langle D \mathcal{L}_{X} \psi, \psi\right\rangle & =\int_{M}\left\langle\mathcal{L}_{X} \psi, D \psi\right\rangle+\int_{\partial M}\left\langle\nu \cdot \mathcal{L}_{X} \psi, \psi\right\rangle \\
& =\int_{M} H \underbrace{\left\langle\mathcal{L}_{X} \psi,|\psi|^{p-2} \psi\right\rangle}_{=\frac{1}{p} \partial_{X}|\psi|^{p}}+\int_{\partial M}\left\langle\nu \cdot \mathcal{L}_{X} \psi, \psi\right\rangle .
\end{aligned}
$$

This yields

$$
\int_{\partial M}\left\langle\nu \cdot \mathcal{L}_{X} \psi, \psi\right\rangle=\frac{p-2}{p} \int_{M} H \partial_{X}|\psi|^{p}+\int_{M} H \beta|\psi|^{p}+\int_{M}\left(\partial_{X} H\right)|\psi|^{p} .
$$

Using $\operatorname{div}\left(H|\psi|^{p} X\right)=\left(\partial_{X} H\right)|\psi|^{p}+H \partial_{X}|\psi|^{p}+H|\psi|^{p} \operatorname{div} X$ and $\operatorname{div} X=n \beta$ we obtain

$$
\begin{aligned}
\int_{\partial M}\left\langle\nu \cdot \mathcal{L}_{X} \psi, \psi\right\rangle= & \frac{p-2}{p} \int_{\partial M} H|\psi|^{p} g(X, \nu) \\
& +\left(\frac{1}{n}-\frac{p-2}{p}\right) \int_{M} H \operatorname{div} X|\psi|^{p}+\frac{2}{p} \int_{M}\left(\partial_{X} H\right)|\psi|^{p} .
\end{aligned}
$$


Examples 3. 1). Let $\Omega$ be a domain in $\mathbb{R}^{n}$ with smooth boundary, let $X=r \partial_{r}=$ $\sum x^{i} \partial_{i}$, and we will assume that $H=\lambda$ is constant. Then $\beta \equiv 1$ and we obtain

$$
\int_{\partial \Omega}\left\langle\nu \cdot \mathcal{L}_{X} \psi, \psi\right\rangle=\lambda \frac{p-2}{p} \int_{\partial \Omega}\langle X, \nu\rangle|\psi|^{p}+\lambda\left(1-\frac{p-2}{p} n\right) \int_{\Omega}|\psi|^{p} .
$$

This inequality bears many analogies to equation (11). In particular, the constant $1-\frac{p-2}{p}$ before the integral over $\Omega$ vanishes if $p$ takes the value $p=2 n /(n-1)$. This value plays the same role in non-linear Dirac equations as the value $p=2 n /(n-2)$ does for the Laplace operator.

2 ). If $M$ is a closed manifold and $X$ is a conformal vector field, then for $p=$ $2 n /(n-1)$ we obtain

$$
\int_{M}\left(\partial_{X} H\right)|\psi|^{p}=0
$$

Corollary 4 (Kazdan-Warner type obstructions). Let $f: S^{n} \rightarrow \mathbb{R}^{n+1}, n \geq 2$, be a conformal immersion (possibly with branching points of even order in the case $n=2)$. We denote by $H: S^{n} \rightarrow \mathbb{R}$ the mean curvature of this immersion. Then, for any conformal vector field $X$ we have

$$
\int_{S^{n}}\left(\partial_{X} H\right) f^{*}(d \mu)=0
$$

where $d \mu$ is the volume element on $f\left(S^{n}\right)$ induced from the Euclidean metric on $\mathbb{R}^{n+1}$. In particular, $\partial_{X} H$ changes sign.

The corollary is particularly interesting in dimension $n=2$. If $f: S^{2} \rightarrow \mathbb{R}^{3}$ is any immersion, then after possibly composing with a diffeomorphism $S^{2} \rightarrow S^{2}$, we can assume that $f$ is conformal.

The corollary is analogous to results in KW75, BE87 and DR99.

Proof. Let $\psi$ be a parallel spinor on $\mathbb{R}^{n+1}$. Then, as proven in KS96, Ba98, Fr98, the restriction of $\psi$ on $\Sigma$ satisfies equation (2) with $p=2 n /(n-1)$, and $|\psi|^{p} d \nu=$ $f^{*}(d \mu)$ where $d \nu$ is the standard volume element on $S^{n}$. Since this equation is conformally invariant we obtain a solution of (2) on $S^{n}$ equipped with the standard metric. The corollary then immediately follows from example (2) above.

Example 5. Let $x_{3}: S^{2} \rightarrow \mathbb{R}$ be the third component of the standard inclusion. One shows that $X:=\operatorname{grad} x_{3}$ is a conformal vector field on $S^{2}$, where the gradient is taken with respect to the standard metric on $S^{2}$. Then for any $\varepsilon \in \mathbb{R} \backslash\{0\}$ one has $\partial_{X}\left(\varepsilon x_{3}+1\right)=\varepsilon g\left(\operatorname{grad} x_{3}, \operatorname{grad} x_{3}\right)$, which is of constant sign. Hence $\varepsilon x_{3}+1: S^{2} \rightarrow \mathbb{R}$ is not the mean curvature of a conformal immersion.

\section{REFERENCES}

[Ba98] C. Bär, Extrinsic bounds for eigenvalues of the Dirac operator, Ann. Global Anal. Geom., 16 (1998), 573-596. MR 1651379 (99k:58183)

[BE87] J.-P. Bourguignon and J.-P. Ezin. Scalar curvature functions in a conformal class of metrics and conformal transformations. Trans. Amer. Math. Soc., 301 (1987), 723-736. MR0882712 (88e:53054)

[BG92] J.-P. Bourguignon and P. Gauduchon, Spineurs, opérateurs de Dirac et variations de métriques, Commun. Math. Phys., 144 (1992), 581-599.. MR.1158762 (93h:58164)

[DR99] O. Druet and F. Robert, On the equivariance of the Kazdan-Warner and the Pohozaev identities, Preprint, 1999.

[Fr98] T. Friedrich, On the spinor representation of surfaces in Euclidean 3-space, J. Geom. Phys., 28 (1998), 143-157. MR1653146|(99i:53057) 
[Fri00] T. Friedrich, Dirac operators in Riemannian geometry, Graduate Studies in Mathematics, vol. 25, Amer. Math. Soc., Providence, RI, 2000. MR.1777332 (2001c:58017)

[Hi86] O. Hijazi, A conformal lower bound for the smallest eigenvalue of the Dirac operator and Killing spinors, Comm. Math. Phys., 104 (1986), 151-162. MR0834486 (87j:58096)

[Ht74] N. Hitchin, Harmonic spinors, Adv. Math., 14 (1974), 1-55. MR0358873 (50:11332)

[KW75] J. Kazdan and F. Warner, Scalar curvature and conformal deformation of Riemannian structure, J. of Diff. Geom., 10 (1975), 113-134. MR0365409 (51:1661)

[KS96] R. Kusner and N. Schmitt, The spinor representation of surfaces in space, Preprint, http://www.arxiv.org/abs/dg-ga/9610005, 1996.

[Po65] S.I. Pohozaev, Eigenfunctions of the equation $\Delta u+\lambda f(u)=0$, Soviet Math. Dokl., 6 (1965), 1408-1411 (English). Translation of Dokl. Akad. Nauk SSSR, 165 (1965), 36-39 (Russian). MR0192184 (33:411)

Institut Élie Cartan, BP 239, Université de NAnCy 1, 54506 Vandoeuvre-Lès-Nancy Cedex, France

E-mail address: ammann@iecn.u-nancy.fr

Institut Élie Cartan, BP 239, Université de NAnCy 1, 54506 Vandoeuvre-LÈs-Nancy Cedex, France

E-mail address: humbert@iecn.u-nancy.fr

Mathematisches Institut der Universität TüBingen, Auf Der Morgenstelle 10, 72076 Tübingen, Germany

E-mail address: ahmedou@analysis.mathematik.uni-tuebingen.de 